\title{
Design an evaluation of RoboCup humanoid goalie
}

\author{
Juan F. García, Francisco J. Rodríguez, Camino Fernández, and Vicente Matellán
}

\begin{abstract}
In this article we describe the ethological inspired architecture we have developed and how it has been used to implement a humanoid goalkeeper according to the regulations of the two-legged Standard Platform League of the RoboCup Federation. We present relevant concepts borrowed from ethology that we have successfully used for generating autonomous behaviours in mobile robotics, such as the use of ethograms in robotic pets or the ideas of schemata, or the use of fixed actions patterns to implement reactivity. Then we discuss the implementation of this architecture on the Nao biped robot. Finally, we propose a method for its evaluation and validation and analyse the results obtained during RoboCup real competition, which allowed us to test first hand how it worked in a real environment.
\end{abstract}

Index Terms-reactive, vision, humanoid, schema

\section{ROBOTICS CONTROL ARCHITECTURES IN LITERATURE}

G ENERATING autonomous behaviours in mobile robotics is really a complex problem. In this section we present a non in-depth outline about those robotics control architectures close to our research. We are going to be neither exhaustive, mainly because it would be impossible to describe all control architectures in just one section, nor hierarchycal, since there would be too many criteria to take into account.

Summarising the complex history of the AI, we can state that two main schools of thought have coexisted, the subsymbolic one, interested on modeling intelligence in a level similar to neurons; and the symbolic AI, which models knowledge and planning in data structures that make sense to the programmers that build them. Another way of explaining the difference between both schools is referring to their foundations: Biology in the subsymbolic AI, and cognitive psychology in the symbolic AI [9]. Hybrid systems are a pragmatic approach, where ethology based systems can be included because they successfully integrate deliberative and reactive perspectives in natural autonomous systems.

Hybrid architectures intend to combine reactive and deliberative control, and usually consist of three components: a reactive layer, a planner, and a layer that links the other two. Well known examples of this kind of architecture are AuRA[1], which integrates a $A^{*}$ planner with schema-based controllers [2], and PRS (Procedural Reasoning System) [6] based on least commitment via plan elaboration postponement.

Teleo-Reactive (TR) program formalism proposed by Nilsson [27] falls also under Hybrid control category. Teleoreactivity in dynamic environments implies a short sense - act cycle. Robots are able react rapidly to commonly occurring situations (such as crash avoidance or refuelling) but their behaviours are also influenced by their goals (hence "teleo").

Juan, Francisco, Camino, and Vicente are with Departamento de Ingeniería Mecánica, Informática y Aeroespacial Escuela de Ingenierías Industrial e Informática, Universidad de León, 24071 León Web: http://robotica.unileon.es e-mail:\{jfgars, fjrodl, camino.fernandez, vicente.matellan $\} @$ unileon.es
Opportunistic architectures are a subset of the hybrid architectures that take its name from Barbara Hayes-Roth approach to hybrid control [7]. The agents architecture on her system was also made up by three components: an event-triggered reactive level, an strategic planner, and a control process in charge of matching triggered actions with the generated plan. A similar architecture is used in O-Plan [8] where the term "agent" is used to name each of the three modules of the system.

Another implementation of these ideas are RAPs (Reactive Action Packages) proposed by Firby [5]. RAPs were designed to allow the reactive execution of symbolic plans. In this way, each RAP defines different alternatives of execution depending on the environment, and an agenda is used to select the next action to execute. Another approach is the TCA (Task Control Architecture) by Simmons [17], which integrates symbolic plans with real-time restrictions as well as reactive behaviours triggered as exceptions.

In the RoboCup domain, Saffiotti [20] presented the ThinkingCap architecture. This architecture was based in a fuzzy approach, extended in [23]. The perceptual and global modelling components managed information in a fuzzy way and were used to generate the next actions.

Also in the RoboCup domain, the architecture proposed by Manuela Veloso et al[21] shows an hybrid hierarchical behaviour-based architecture. This architecture was divided in levels. The upper levels set goals that the bottom level had to achieve using information generated by a set of virtual sensors, which were an abstraction of the actual sensors.

Another successful approach in the RoboCup was the one used in the German Team[22] that proposed a four levels architecture: perception, object modelling, behaviour control, and motion control. The execution starts in the upper level perceiving the environment and finishes at low level sending motion commands to actuators. The behaviour level was made up of several basic behaviours implemented as finite state machines. These finite state machine were written in XABSL language [24], that was interpreted at runtime and let change and reload the behaviour during the robot operation.

Many other concepts borrowed from Ethology have been used in robotics. For instance, homoeostasis, proposed as mechanisms for action selection by T. Tyrrell [15]; or the flies balancing optical flow in both eyes to local navigation [4]; gestalt perception, and the use of visual perceptive invariants, as the ones discovered in the cormorant fishing [14], that can make easier the goal of developing robotic behaviours, etc. These works have also been applied to modern humanoids [3].

The foundation of the work presented in this paper is JDE (Jerarquía Dinámica de Esquemas) [9], an etho-inspired architecture where behaviour is organised as a dynamic hierarchy of 
independent schemata. This architecture is hybrid in nature, so it is closely related to the other hybrid approaches previously enumerated.

Besides theoretically describing the architecture, we have also implemented it in a robot in order to put it to the test in a real environment. The chosen scenario was the RoboCup (Robotic soccer WorldCup), an international research and education initiative, which has put forward a standard problem to promote the research on artificial intelligence and intelligent robotics.

In particular, the work described in this paper has been tested in the Standard Platform League (SPL) during German Open $2009^{1}$ and Robocup $2009^{2}$. In this league, all teams use the same hardware platform, the Nao robot (see figure 1). These robots are manufactured by Aldebaran Robotics, so the focus of this competition is on the software controlling the robot.

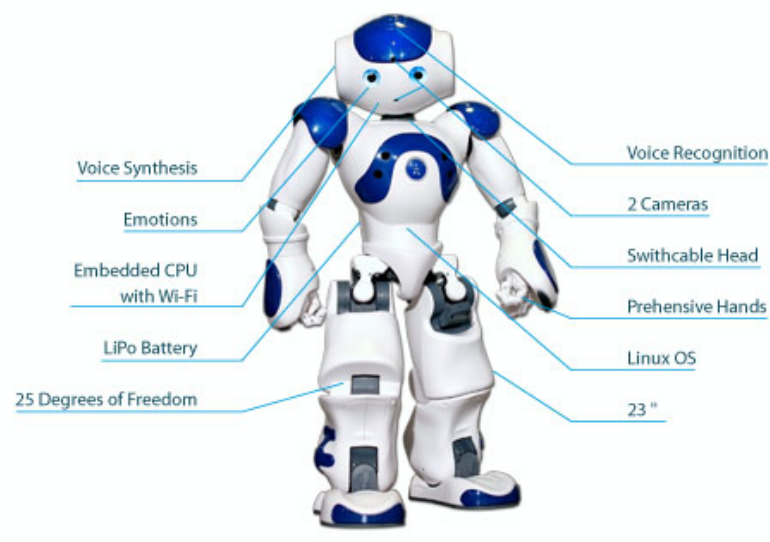

Fig. 1. Nao robot (figure copyrighted by Aldebaran Robotics)

Nao robot is a 21 degrees of freedom humanoid, whose height is $57 \mathrm{~cm}$. and its weight is around $4.5 \mathrm{Kg}$. It has two 30 fps video cameras located in the forehead and in the mouth, each one with a maximum resolution of $640 \times 480$, but they cannot be used simultaneously. The switch between cameras takes too long and the field of view is scarcely overlapped so they are not capable of stereo vision.

Control is managed on-board using a x86 AMD Geode chip at $500 \mathrm{MHz}, 256 \mathrm{MB}$ of SDRAM memory and $1 \mathrm{~Gb}$ of flash memory that can be upgraded. It also has got WiFi (802.11g) and Ethernet connections. Concerning the sensors, apart from the cameras, it has 2 gyroscopes and 3 accelerometers, 2 bumper sensors in the feet, and 2 ultrasonic sensors in the chest.

The rest of the paper is organised as follows. Second section describes the architecture we propose. In the third section we present a software implementation for our architecture. In the fourth section, we propose a method to analyse and validate our proposal. Finally, in the last section, the results obtained with this architecture and its performance in the RoboCup

\footnotetext{
${ }^{1}$ http://www.robocup-german-open.de/en

${ }^{2}$ http://www.robocup2009.org/
}

German Open and in the Robocup 2009 Graz are analysed and also future works are enumerated.

\section{AN ETHOLOGICAL INSPIRED ARCHITECTURE}

Our architecture is based on ethological principles that exhibit the same features of the hybrid ones previously described, that is, deliberative and reactive capabilities. The two main principles of this architecture are the decomposition of the control problem into behavioural units named components, and the generation of behaviour by building dynamic hierarchies. Both are detailed in next sections.

\section{A. Components}

Our approach is based on the assumption that complex behaviour can be obtained by combining simpler "components" inspired by ethological schemata as defined in [10]. These components perform a specific task in an iterative way and at a controlled frequency. They may send commands to actuators, process data from sensors, or activate/deactivate and modulate other components creating a hierarchy.

When activated, a component creates its data and processing structures and starts its iterations. It can keep its state from one iteration to another or change it depending on its functionality and the system stimuli (internal or environmental information). When a component is deactivated, all its descendants (all the components the currently component becoming inactive had activated) must also be finished.

A group of components which perform subroutines of the same task are grouped in so called controllers which functionality is explained in section II-C and their implementation in section III.

\section{B. Dynamic Hierarchy}

Components are organised in hierarchy in order to generate more complex behaviours. High level components activate low level components, and all of them run concurrently. The hierarchy is dynamic in the sense that currently active modules will be different depending on the situation.

All schemata in the same level are mutually exclusive, which means only one schema per level can be active at any given time. Also, before activating any component, an ancestor of it in the immediately superior level has to be already active. First restriction helps minimising the risk of trying to perform contradictory tasks, improving system stability: for instance, "move" and "save" schemata are in the same level and both of them send commands to the robot's actuators and servos; if they were activated simultaneously, the result of combining these commands would be unpredictable, and the robot would probably fall. Second restriction guarantees that all prerequisites for the task to be performed are fulfilled before activating the component.

Upon deactivating a component, all its descendants will also become inactive. Each schema or a whole branch of linked schemata can be activated (or deactivated) at any given time to achieve the desired functionality, completely deactivating a previously working set of schemata if necessary. 
This makes an improvement to the initial JDE assumption which establishes that every single schema in a certain active hierarchy has to be deactivated one by one before starting a new one.

Components use a common shared memory space to read its inputs and write its outputs. The upper level component connects the output with the inputs of the modules it activates. This way a low level component could be reused by another high level components which could decide to connect the low level components in a different way. All these inputs and outputs define the system information flow, which basically consists of internal (component generated) or external (from the environment) stimuli. All components output are then internal stimuli, while their input can be either an internal or external stimuli depending where it comes from.

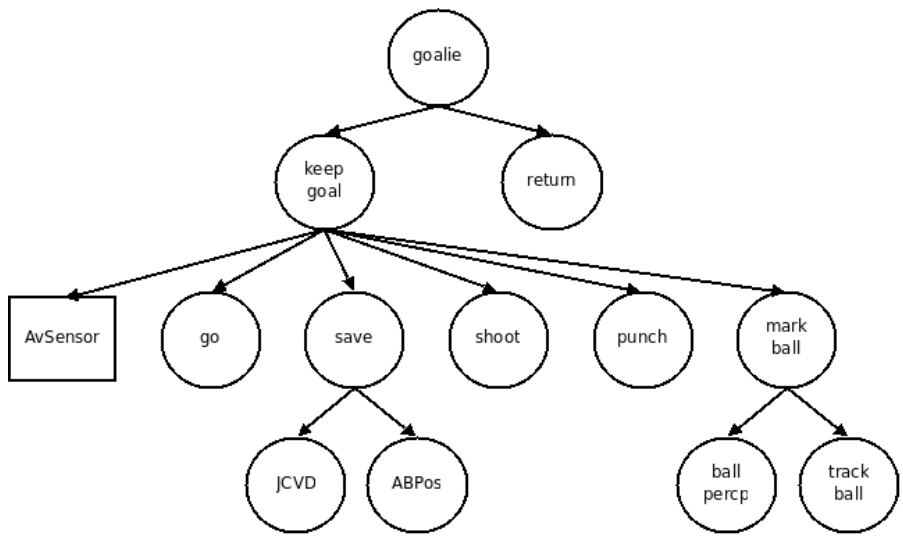

Fig. 2. Goalkeeper modules

Figure 2 shows an example of hierarchy, the goalkeeper behaviour schemata.

\section{Controllers}

As already explained in section II-A, a controller is a group of schemata which perform subroutines of the same task. The components are grouped to simplify the overall structure of the architecture: it is an effective way to reduce the information flow present in the system.

Information, as we explained in the previous section, consists of external or system internal stimuli which would cause either activation or deactivation of a given component. The main reason to have controllers and not individual ungroupped components is not having to consider an input and output information channel per component of the system at any given time. Instead, information is brought to each controller, and it will then be redirected to the concrete component which is designed to react to it.

Basically, a controller oversees the activation and deactivation of its components redirecting the information flow it receives and produces. Each controller is able to communicate with other controllers coexisting in the system to which it is directly connected the same way isolated components do.

Besides the conceptual simplicity explained, there is no real difference among a controller and a group of components. We describe the controllers we use, its functionality , and its implementation in section III.

\section{Architecture characterisation}

The presented architecture shows both deliberative and reactive properties, so it is a hybrid architecture in the classic definition. The set of all possible connections among components and their organisation in different levels, as shown in figure 2, give the system its deliberative nature. In this figure, circles are components, while squares represent system's sensors which provide inputs from the environment (in this case, only the camera sensor is shown). The higher the level, the more abstract and complex behaviours it contains.

The architecture is reactive during the hierarchy activation phase previously explained in II-B: the set of active components will vary depending on the situation, with only those useful for the current behaviour being active. We will give two examples of its reactive nature in next section.

Please note that even if the hierarchy defined by active schemata in a given situation is dynamic (varies depending on the task at hand) - reactive behaviour - the available connections among components and their organisation in levels is fixed and previously established - deliberative architecture -.

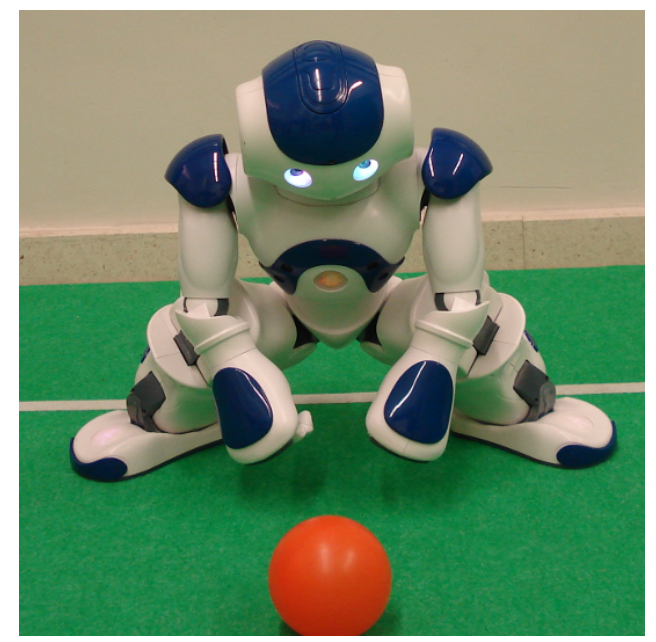

Fig. 3. JCVD defensive movement schema implemented on real Nao robot

\section{IMPLEMENTATION}

We are interested in testing our architecture in order to prove its functionality. To do so we have chosen to model a goalkeeper behaviour.

\section{A. Components}

The components are the ethological schemata which model all the possible actions the goalkeeper needs. We have the following components (with each component's name being pretty much self-explanatory about their functionality):

- Goalie: Represents all kind of high level behavioural decisions which a goalkeeper would perform during a match, either specific to its role (eg: punch out the ball), or not (perception tasks).

- KeepGoal: Represents all kind of high level behavioural decisions which are specific to a goalkeeper's role. 
- MarkBall: Tries to keep the ball inside the robots visual field.

- BallPerception: Looks for the ball in a given image.

- TrackBall: Moves the robots head so that the ball stays in the centre of its visual field.

- Go: Makes the robot walk to a given position.

- Return: Makes the robot walk to the centre of its keep.

- Save: Performs a defensive move to try to stop the ball.

- JCVD: A wide-area but slow defensive move intended to prevent a goal.

- ABPos: A fast but small-area defensive move intended to prevent a goal.

- Shoot: Performs a kick to clear the ball.

- PunchOut: Punches out the ball.

\section{B. Controllers and NaoQi Layer}

Task related components are grouped into controllers. The controllers we have implemented, its main functionality, and the components they include are:

- Goalkeeper Controller: Takes high level decisions about what to do at any given time: look for the ball, move or try to prevent a goal. Includes Goalie and KeepGoal components.

- Scanner Controller: Moves the robot head in order to look for the ball and gets and analyses images from the robot's camera. Includes MarkBall, BallPerception and TrackBall components.

- Walk Controller: Allows the robot to walk in different directions. Includes Go and Return components.

- Save Controller: Performs defensive positions intended to stop or clear the ball. Includes Save, JCVD, ABPos, Shoot and PunchOut components.

Those controllers have been used, as already introduced in section II-C, to reduce the system complexity. By grouping components which take part in the same task we reduce the amount of information channels to be considered at any given time. For instance: BallPerception and TrackBall components are meant to work with visual information (the first one will look for the ball in any image obtained by the robot camera while the second one will try to centre it in the field of view once it has been found). There is no reason then to use two different information channels carrying the same visual information, so we group them in a controller which we call Scanner Controller which will receive this information and then redirect it to the component which actually needs it.

To be able to control the robot, we will use the software it provides, called NaoQi. NaoQi is a proprietary SDK which allows us to access robot sensors and actuators by using the modules it provides. We can not consider that NaoQi modules define a real controller since they implement very different functions, from internal memory management to servo motors control, and thus are not really task-related. However, for simplicity reasons, we will represent all these modules together grouped in what we call NaoQi Layer. Also, the only conceptual difference between NaoQi modules and the rest of our components is that components in the NaoQi layer are platform specific, that is, they are part of Nao robot's software.
The hierarchical relation between all the controllers can be seen in figure 4.

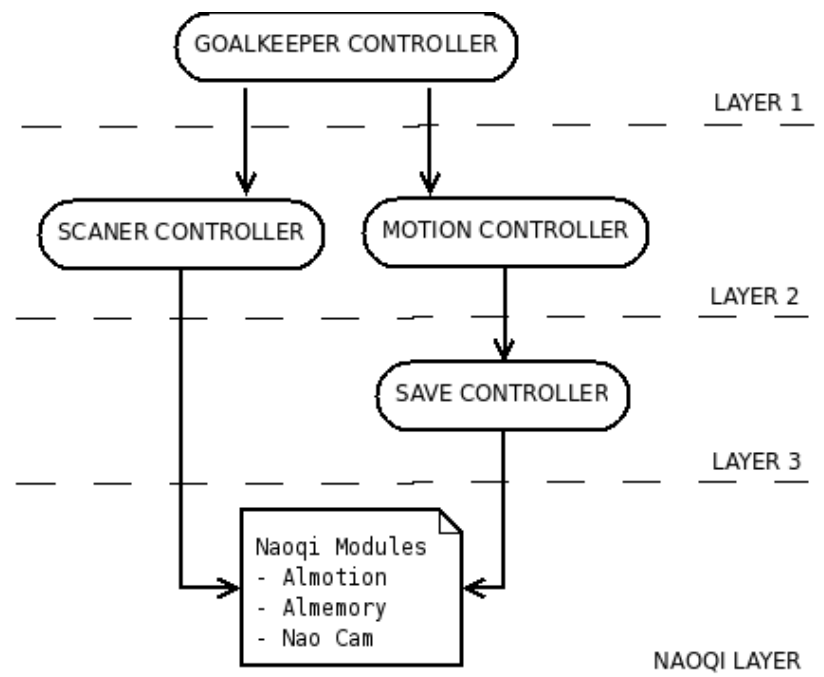

Fig. 4. Goalkeeper controllers

\section{Hierarchy}

The whole static hierarchy, which consists of all implemented components, can be seen in figure 2 , and the controllers which they are part of are shown in figure 4 . This static hierarchy represents the deliberative nature of the architecture. The components are organised in levels, with those related to high level tasks occupying the top ones while other more being in the lower levels. The lines connecting components represent the hierarchical relation between them.

The hybrid nature of our architecture can be better illustrated by two examples of generation of autonomous behaviour for our RoboCup goalkeeper.

Example I. Let's assume only scanning and basic saving positions modules are available during a real match (deliberative architecture). Given this situation, the goalkeeper would just activate the TrackBall component (after activating the needed ascendant schemata to reach it) - reactive architecture - (see figure 2), thus activating KeepGoal and MarkBall on its way down to it. It would also eventually try to stop it if it comes too close to the keep by going down the hierarchy activating KeepGoal, Save and finally ABPos (a static defensive position)..

Example II. Let's suppose all schemata are available during the match. In this case, the goalie, once the ball has been found, would perform side steps to position itself in front of the ball, activating Keep_goal and go schemata. It could even clear the ball activating the punch-out component (which is also connected to Keep_goal) if the ball comes close enough.

We have two videos ${ }^{34}$ in our web that show these examples working in a real Nao humanoid. Both videos show the whole tree of components, with the active components displayed in a lighter color. The Nao robot appears to the left of the media

\footnotetext{
${ }^{3}$ http://robotica.unileon.es/mediawiki/videos/save.swf

${ }^{4}$ http://robotica.unileon.es/mediawiki/videos/movementAndSave.swf
} 
player, performing each action enumerated in both scenarios, while we can observe its internal architecture displayed at the right side. The relation among components and the dynamic hierarchy resulting from their synchronisation are also represented: some schemata are activated when required by the situation while others no longer necessary become inactive.

\section{Real world restrictions}

When implementing our architecture in a real robot some issues were raised. For instance, not all actions can be instantly stopped (specially those related to movement) to start a new one. This affects not only robots also humans: just imagine you are running and suddenly decide to lay on the floor; you better slow down and stop moving before trying to do so or you will end up rolling on the floor. Applied to our component based design, this means component deactivation will always have a time cost. It is not possible to model this cost because it depends on the current situation.

In most cases, deactivation times are so brief that can be ignored, such is the case of decision-related components like KeepGoal, Save, MarkBall or TrackBall (not much time is necessary to decide you are no longer interested in defending your keep or tracking the ball). However, time cost for movement-related ones like Go, ABPos or JCVD (see figure 2) are not negligible, as shown by figure 5. Although these time costs may seem too high, imagine for a moment the time it would take to a human to stop running, fall to the floor to try to stop the ball, and then get up and start running again.

We need to perform some actions before fully deactivating any of the movement-related components so that the robot is not left in an unstable position and thus becomes suitable to fall. We could consider that some sort of cooldown timer is set preventing any new schema activation until the last deactivated schema ensures the robot has reached a stable state. As a result, all schemata have an associated deactivation cost.

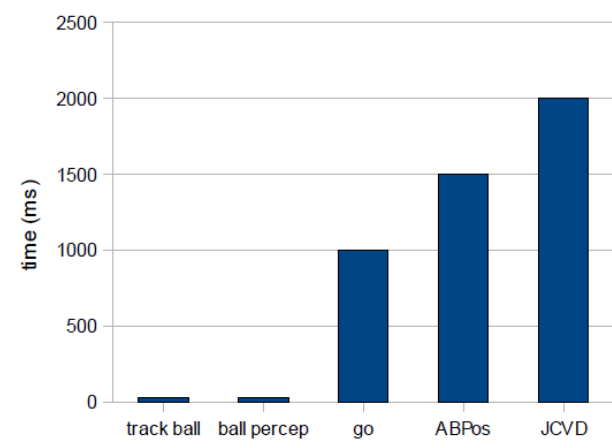

Fig. 5. Deactivation time cost for some schemata

Taking these times into account, some sort of high level deliberative mechanism is necessary for behaviour planning: It is necessary to evaluate the advantages (goal achieved) and drawbacks (in terms of time cost) to deactivate a schema in order to activate a new one. Should I really stop running to comb my hair if I am running to try to catch the bus?, should I stop running to tie my shoes if I am, again, trying not to lose the bus? The answer to first question is obviously "no" since my goal is to get in time before the bus leaves, but it would probably be "yes" to the second one, since it may not be worth taking the risk of falling.

In the robotics world, and specifically in the Robocup environment, we also have plenty of situation which illustrate this kind of situations. For instance, imagine the robot is moving sideways (see figure $6,(1)$ ). When close enough to the ball, the Goalkeeper Controller decides it should stop and try to block it by using a fast defensive move (ABPos or $J C V D)$. The Go schema should be deactivated, which would consume a second (see figure 5). As soon as go is inactive, Save is activated, and then JCVD becomes active too (labelled as (2) in figure 6). If the ball suddenly moves away from the goalie (for whatever reason), it will have to stand up and move again. JCVD schema would be deactivated, which would consume 2 seconds (it takes some time to get up from the floor). Then, Save schema would get inactive (barely instantly since it is just a decision related schema) and finally go could be reactivated (see (1) in the same figure). So basically, a "move - stop - save - get up - move" sequence would take more than 3 seconds to be performed in reality (without taking into account the time the save would take per-se), while theoretically those times were neglected.

\section{ARCHITECTURE ANALYSIS AND VALIDATION}

For the Standard Platform League, with the Naos being a relatively new addition, the level of play of many teams is not yet that sophisticated. Our goalkeeper was not called upon to save many goals and so it is difficult to assess its effectiveness relaying just on the results obtained during the championship. It is also difficulty to assess how well would the robot perform without this architecture. Keeping these limitations in mind, we are trying to evaluate the architecture the most objective way possible. To do so, we review all levels presented in the "4+1 View Model of Architecture" by Krutchen [25], using the norm ISO 9126, an international standard for software quality evaluation:

1) Logic level. High level programming allowed by schemata and behaviour units usage and low level details being hidden thanks to NaoQi both improve abstraction. The architecture makes it easier to understand already developed behaviours and actions and simplyfies the process of adding new ones, so it complies with "usability" characteristic of ISO 9126. Although it doesn't directly guarantees efficiency, it makes it easier to achieve it since every module can be tested and improved its own.

2) Processes level. The goalkeeper behaviour is split in different levels, with every level performing actions independently form the rest (movement, vision, etc.). This makes concurrency management much easier and at the same time it simplyfies the process of adding a new behaviour (for instance, a new kick o new scan mode) or even a whole new controller (for instance, a localization controller) without interfering with the already existing ones. ISO 9126 "Security", "interoperability", and "sta- 


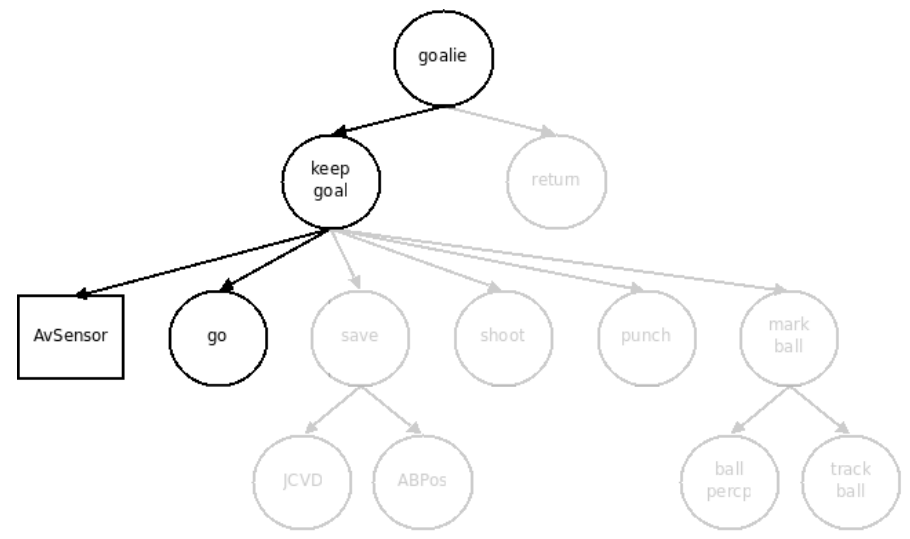

(1)

Fig. 6. Schemata active during a "move (1) - save (2) - move (1)" sequence

bility" categories are then maximised when using this architecture.

3) Development level. To evaluate development advantages, that is, how this architecture makes the programming of the Nao easier or how behaviours are more quickly developed when using it, we suggest using cocomo (COnstructive COst MOdel), a mathematical empirical model for software costs estimation [26].

4) Hardware. The most interesting aspect of our architecture about hardware is that all platform specific calls and functions are contained inside NaoQi layer. Since this layer is developed and maintained by the Nao's company (Aldebaran), hardware optimised usage is taken outside of the architecture and solely relies on their external development. The existence of the NaoQi layer also means that we could use this very same implementation except for that layer for any other robot, which complies with the "potability" category of ISO 9126.

5) Performance of the four previous levels when working together. The best way to evaluate performance of architecture as a whole is put it to the test. For that reason, in section IV-A a battery of goalkeeper specific tests is proposed. The matches played during Hannover German Open and Graz Robocup are also a good benchmark themselves.

\section{A. Goalkeeper behaviour specific tests}

The architecture described has been used to model a goalkeeper behaviour. The best way to test its elements is to check how well a robot with an implementation of it performs the goalkeeper rol. The most relevant tasks fulfilled by a goalkeeper are then put to the test: fast movement over the field, ball perception, ball tracking, proper positioning (deliberative part), and goal saving (reactive part). Since movement speed, and movement in general, is dependant on NaoQi primitives, as it was previously stated in section III-B, it is not taken into account. In order to test the rest of them, the following benchmark is proposed:

Only one side of the field is used. A set of markers are placed on it: they are placed at 4 rows and 5 columns, first

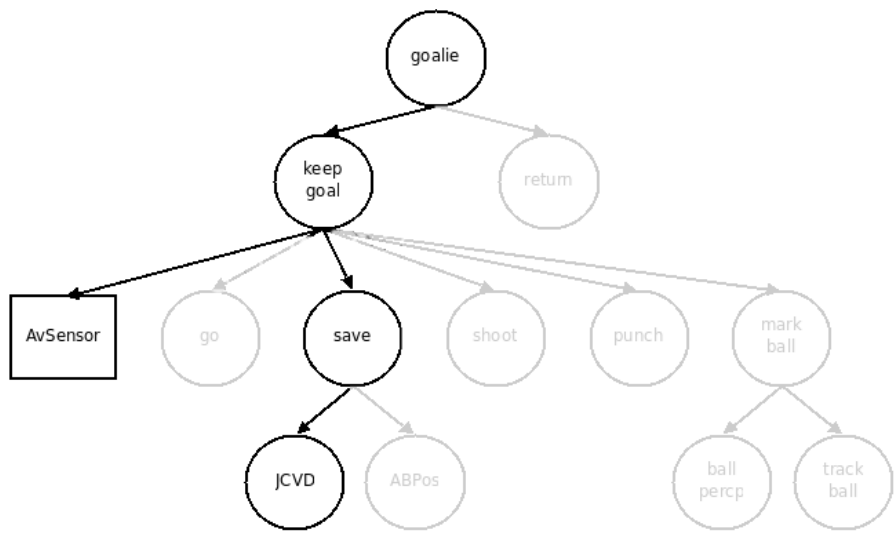

(2)

row at $50 \mathrm{~cm}$ from the keep line and the rest $50 \mathrm{~cm}$ from the previous one. The markers are labelled as $(m, n)$, with $m$ (rows) ranging from 0 to 3 and $n$ (columns) from 0 to 4, with marker $(0,0)$ being the one at the top-left corner when looking at the keep and marker $(0,2)$ being positioned exactly in front of the robot, at $50 \mathrm{~cm}$ from the keep's line. All markers in the same row are also positioned $50 \mathrm{~cm}$ from the adjacent ones. Fig. 7 shows a top view of the benchmark proposed.

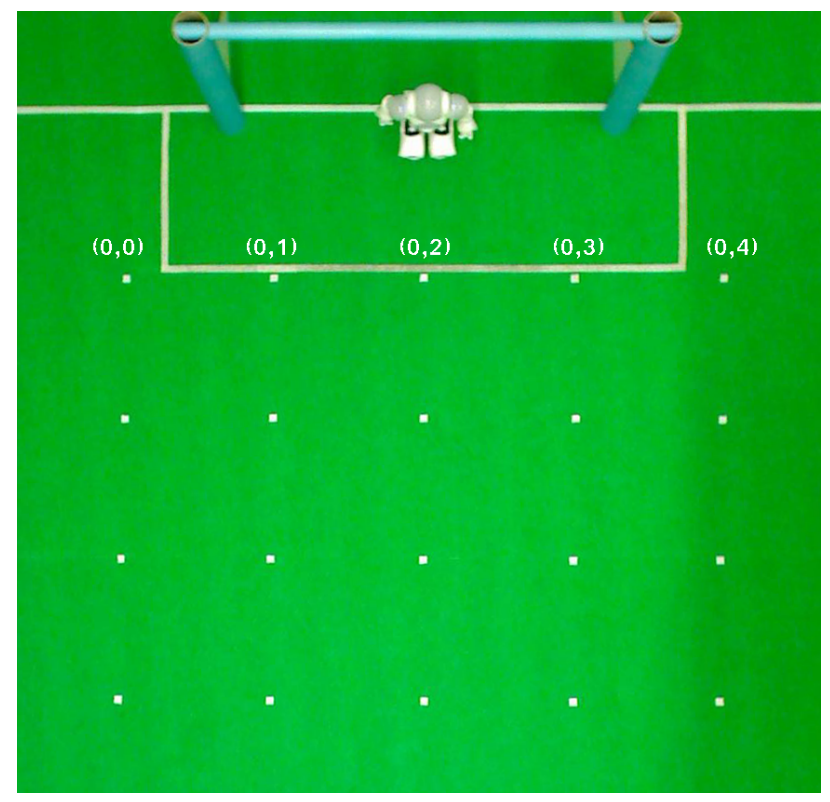

Fig. 7. Markers used for testing

A $130 \mathrm{~cm}$ length ramp with variable inclination from $6 \%$ to $24 \%$ is used to perform the test. The ball can be placed on three different positions on top of it, at 50,100 and $130 \mathrm{~cm}$ from its lower part, which is placed on every marker in the field. For every marker, the angle to the keep can be modified from $-\frac{\pi}{4}$ rad to $\frac{\pi}{4} \mathrm{rad}$ with steps of $\frac{\pi}{16} \mathrm{rad}$, which results in nine possible orientation for every marker: $\left\{-\frac{\pi}{4},-\frac{5 \pi}{16},-\frac{\pi}{8},-\frac{\pi}{16}, 0, \frac{\pi}{16}, \frac{\pi}{8}\right.$, $\left.\frac{5 \pi}{16}, \frac{\pi}{4}\right\}$. This allows us to test nine different orientations and three different ball starting position for every marker; since twenty markers are placed in the field, it means the benchmark 
includes a total of 540 different shots. Shot speed can also be modified depending on the ramp's inclination.

To perform the test, the goalkeeper stands in the middle of the keep when started. The ramp is then positioned at every marker (different ball positions on top of it, and different angles and inclination are used for all of them). Once the goalkeeper locates the ball and starts tracking it, the ball is realeased from its initial position and thus approaches the keep. For every shot, the goalkeeper success rate in every category evaluated (ball perception, ball tracking, proper positioning, and goal saving) is measured.

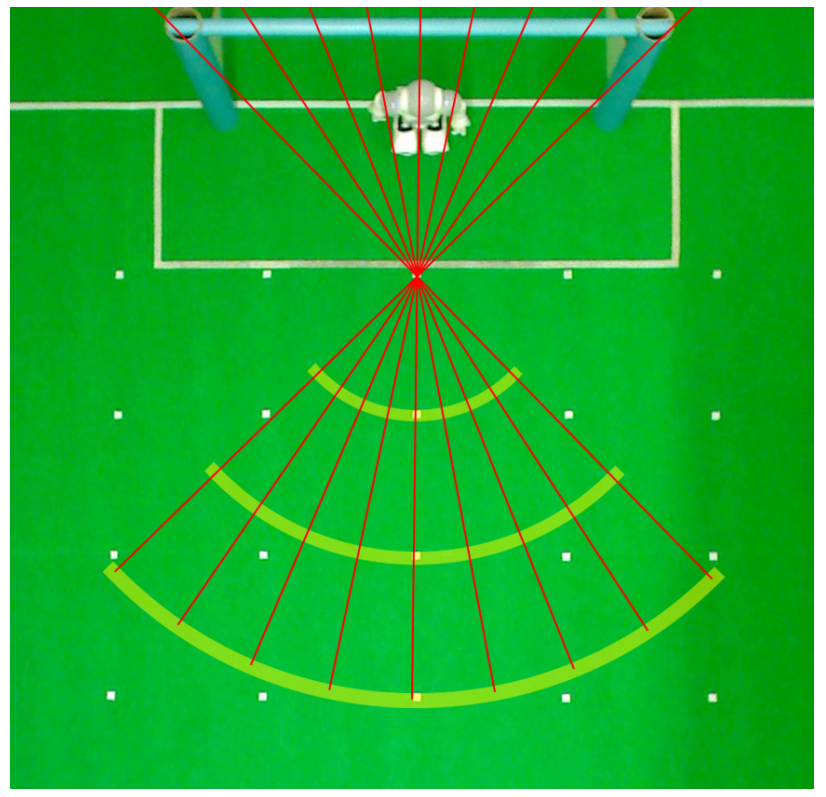

Fig. 8. Possible orientations from marker $(0,2)$

To evaluate the performance of the goalkeeper, only markers $(0, n)$ from first row were used to test the architecture. Ramp inclination was also fixed at $6 \%$. All nine possible angles and the three starting ball positions on top of the ramp were used. This means 135 different shots were used to test our behaviour. Every shot was repeated ten times to ensure more reliable results, which gives us a total of 1350 measures. Fig. 8 shows the different trajectories possible from marker $(0,2)$ and Table I shows the success rates (in \%) obtained for every characteristic measured and every test performed. Ball perception and tracking is almost flawless, and positioning is also good, specially for central and side markers, that is, $(0,0),(0,2)$, and $(0,4)$. Positioning being not very accurate for markers $(0,1)$ and $(0,3)$ (see Fig. 7 ) is a consequence of far post shots from these positions: the goalkeeper tries to always position itself in front of the ball, which is better to intercept shots aimed at the near post but worse for far post shots, which mostly end up in a goal.

\section{CONCLUSIONS AND FURTHER WORK}

In this paper we have presented a hierarchical architecture, borrowing concepts such as components (schemata) or dynamic hierarchies from ethology and adding others like the controller concept to simplify the architecture design and the information flow inside the system.

This architecture has been implemented on the software infrastructure offered by the NaoQi development environment for the Nao humanoid.

The implementation has been evaluated using the " $4+1$ View Model of Architecture" by Krutchen [25]. To further validate its performance in real gameplay environment, a benchmark of goalkeeper specific behaviours has been proposed. The architecture was also tested during RoboCup German Open (April 2009) and RoboCup Official tournament (July 2009). The results obtained in both the benchmark and the competitions show the correctness of the approach:

- The architecture mantains a goalkeeper behaviour for the whole test or match (20 minutes for the former and 10 minutes for the later, both without human intervention).

- The architecture adapts to dynamic match situations, mainly ball position.

- The architecture implements the goalkeeper behaviour using a deliberative structure for planning (self-positioning) and a reactive control mechanism to respond to environmental and internal stimuli (goal saving).

- The architecture allowed the robot to track the ball nearly $100 \%$ of the time, position itself properly $84 \%$ of the time, and save goals from $62 \%$ of the trajectories tested.

Future works envisioned are improving reactive nature of the system via gaze control implementation to react to other elements apart from the ball, and testing architecture's suitability to more complex behaviours that include collaboration with other robots. Minor improvements in goalkeeper positioning behaviour to make it less vulnerable to far post shooting would also be interesting given the results obtained in the tests.

\section{ACKNOWLEDGEMENT}

We want to express our gratitude and acknowledgement to all members of our team, in particular to the members of the robotics group of the Rey Juan Carlos university for their support, help, and inspiration of the ideas described in this paper.

The authors would like to thank the Spanish Ministry of Innovation for its support to this project under the grant DPI2007-66556-C03-01 (COCOGROM project), and Junta de Castilla y León and European Social Fund for their support to Juan F. Garcia.

\section{REFERENCES}

[1] R. C. Arkin, T. Balch. AuRA: Principles and Practice in Review. Journal of Experimental and Theoretical Artificial Intelligence, 9(2-3), pp. 175188, 1997

[2] R. C. Arkin. Motor Schema-Based Mobile Robot Navigation. The International Journal of Robotics Research, 8(4), pp. 92-112, 1989

[3] S. Chernova, R. C. Arkin. From deliberative to routine behaviours: a cognitively-inspired action selection mechanism for routine behaviour capture. Adaptive Behaviour Journal, Vol. 15, No. 2, pp. 199-216, 2007.

[4] A. P. Duchon, W. H. Harren, L. P. Kaelbling. Ecological robotics. Adaptive Behaviour 6 (3/4), special Issue on Biologically Inspired Models of Spatial Navigation, pp. 473-507, 1998.

[5] R. J. Firby. Task networks for controlling continuous processes. In: Proceedings of the 2nd International Conference on AI Planning Systems AIPS-94. Chicago, IL (USA), pp. 49-54, 1994 


\begin{tabular}{|c|c|c|c|c|}
\hline marker & ball perception & ball tracking & proper positioning & goal saved \\
\hline \hline$(0,0)$ & $100 \%$ & $100 \%$ & $100 \%$ & $78 \%$ \\
\hline$(0,1)$ & $100 \%$ & $97 \%$ & $61 \%$ & $50 \%$ \\
\hline$(0,2)$ & $100 \%$ & $99 \%$ & $98 \%$ & $58 \%$ \\
\hline$(0,3)$ & $100 \%$ & $97 \%$ & $63 \%$ & $54 \%$ \\
\hline$(0,4)$ & $100 \%$ & $100 \%$ & $100 \%$ & $72 \%$ \\
\hline
\end{tabular}

TABLE I

GOALKEEPER'S BEHAVIOUR TEST RESULTS

[6] M. P. Georgef, A. L. Lansky. Reactive Reasoning and Planning, Proceedings of AAAI-87 Sixth National Conference on Artificial Intelligence, Seattle, WA (USA), pp. 677-68, 1987.

[7] B. Hayes-Roth. Opportunistic control of action in intelligent agents, IEEE Transactions on Systems, Man, and Cybernetics, Vol 23:6, pp. 1575-1586, 1992.

[8] K. Currie, A. Tate. O-Plan: The Open Planning Architecture, Artificial Intelligence, Vol. 52:1, pp. 49-86, 1991.

[9] J. M. Cañas, V. Matellán. From Bio-inspired vs. Psycho-inspired to Ethoinspired robots, Robotics and Autonomous Systems, Vol.55, Num. 12, pp. 841-850. DOI:10.1016/j.robot.2007.07.010

[10] J. M. Cañas, J. Ruíz-Ayúcar, C. Agüero, F. Martín. JDE-neoc: component oriented software architecture for robotics, Journal of Physical Agents, Volume 1, Number 1, pp. 1-6, 2007.

[11] J. F. García, F. J. Rodríguez, C. Fernández, V. Matellán. Designing a minimal reactive goalie for the RoboCup SPL. WAF 2009 (Workshop de Agentes Físicos) Cáceres (Spain), 2009.

[12] A. N. Allen, J. C. Shaw, H. A. Simon. Report on a general problemsolving program, Proceedings of the International Conference on Information Processing, pp. 256-264, 1959.

[13] K. Lorenz. Foundations of ethology, Springer Verlag, New York, 1981.

[14] D. McFarland, T. Bösser. Intelligent Behaviour in Animals and Robots, MIT Press, ISBN: 0-262-13293-1, 1993.

[15] T. Tyrrell. An evaluation of Maes' bottom-up mechanism for behaviour selection, Journal of Adaptive Behaviour, Vol. 2, Num 4, pp. 307-348, 1994.

[16] G. Walter. An imitation of life. Scientific American, 1950.

[17] R. Simmons, R. Goodwin, K. Haigh, S. Koenig, J. O'Sullivan, M. Veloso. Xavier: Experience with a Layered Robot Architecture, Agents '97, 1997.

[18] A. Stoytchev, R. C. Arkin. Combining Deliberation, Reactivity, and Motivation in the Context of a Behaviour-Based Robot Architecture, In
Proceedings 2001 IEEE International Symposium on Computational Intelligence in Robotics and Automation. 290-295. Banff, Alberta, Canada, 2000.

[19] R. C. Arkin. Motor Schema Based Mobile Robot Navigation, The International Journal of Robotics Research, Vol. 8, No. 4, pp. 92-112, 1989.

[20] A. Saffiotti, Z. Wasik. Using hierarchical fuzzy behaviours in the RoboCup domain, Autonomous robotic systems: soft computing and hard computing methodologies and applications. pp. 235-262. Physica-Verlag GmbH. Heidelberg, Germany, 2003.

[21] S. Lenser, J. Bruce, M. Veloso. A Modular Hierarchical BehaviourBased Architecture, Lecture Notes in Computer Science. RoboCup 2001: Robot Soccer World Cup V. pp. 79-99. Springer Berlin / Heidelberg, 2002.

[22] T. Rofer, H. Burkhard, O. von Stryk, U. Schwiegelshohn, T. Laue, M. Weber, M. Juengel, D. Gohring, J. Hoffmann, B. Altmeyer, T. Krause, M. Spranger, R. Brunn, M. Dassler, M. Kunz, T. Oberlies, M. Risler, M. Hebbela, W. Nistico, S. Czarnetzkia, T. Kerkhof, M. Meyer, C. Rohde, B. Schmitz, M. Wachter, T. Wegner, C. Zarges. German team: Robocup 2005. Technical report, Germany, 2005.

[23] A. Skarmeta, H. Martínez. Fuzzy Logic Based Intelligent Agents for Reactive Navigation in Autonomous Systems, Fitth International Conference on Fuzzy Theory and Technology, Raleigh (USA), 1997

[24] M. Loetzsch, M. Risler, and M. Jungel. XABSL - A pragmatic approach to behaviour engineering. In Proceedings of the IEEE/RSJ International Conference on Intelligent Robots and Systems (IROS 2006), pp. 51245129, Beijing, October 2006.

[25] P. Kruchten. Architectural Blueprints The "4+1" View Model of Software Architecture, IEEE Software 12 (6), pp. 42-50, November 1995.

[26] B. W. Boehm. Software Engineering Economics, Prentice-Hall, 1981.

[27] N. Nilsson. Teleo-Reactive Programs for Agent Control, Journal of Artificial Intelligence Research, pp. 139-158, 1994. 